\title{
Procedimiento para la caracterización de una celda de punto triple de agua Isotech en el LATU
}

\author{
Robatto, O. ${ }^{(1)}$, Sica, A. ${ }^{(1)}$, Santo, C. (1) \\ Contacto: orobatto@latu.org.uy \\ (1) Departamento de Metrología, Laboratorio Tecnológico del Uruguay (LATU) \\ Recibido: 1/10/2008 - Aprobado: 1/12/2008
}

\begin{abstract}
$\underline{\text { Resumen }}$
La realización en el punto triple de agua es crucial para una mejor aproximación a la Escala Internacional de Temperatura (ITS90), ya que la definición de esta última está directamente vinculada al punto fijo citado. Es necesario conocer la influencia de la presión hidrostática en el punto triple de agua para corregir los valores de resistencia medidos y obtener una mejor aproximación al valor real de la temperatura en dicho punto.

Este artículo describe las dificultades encontradas al estudiar el perfil térmico correspondiente a una celda de punto triple de agua comercial bajo condiciones normales de operación con un baño Dewar termostatizado con hielo picado.

Este trabajo fue llevado a cabo en dos fases: en la primera se detectaron problemas con los perfiles de temperatura, cuyo comportamiento era lejano al ideal. Para mejorar dicho comportamiento se hicieron cambios en el equipamiento, con el fin de minimizar el tiempo de estabilización de la celda y mejorar la transferencia de calor. El aislamiento del baño fue mejorado y se colocó un dispositivo ecualizador de temperatura de cobre en el pozo de la celda. Los resultados se discuten en el presente artículo.

Palabras clave: punto triple de agua, Escala Internacional de Temperatura de 1990 (ITS-90), perfil térmico, presión hidrostática.
\end{abstract}

\begin{abstract}
$\underline{\text { Abstract }}$
The Realization of the Triple Point of Water (TPW) is of main importance when approximating to the International Temperature Scale of 1990 (ITS-90), because it is properly involved in its definition. Therefore it is necessary to know the Hydrostatic Pressure influence at the Triple Point of Water to correct the values of resistance measured and to obtain a better approximation of the real temperature of this point. This article shows the difficulties found when studying the thermal profile of one commercial cell of the triple point of water under normal operation conditions in a Dewar thermostatic bath with crushed ice. The work was performed in two phases. In the first phase problems were detected with the temperature profiles, whose behaviors were far away from the ideals ones. To improve this behavior changes were made in the equipment. The isolation of the bath was improved and copper bushing was set up into the well of the cell. Results are discussed in this paper.

Key words: Triple Point of Water, International Temperature Scale of 1990 (ITS-90), Thermal Profile, Hydrostatic Pressure.
\end{abstract}

\section{Introducción}

Una celda de punto triple de agua es uno de los patrones más importantes para la realización de la Escala Internacional de Temperatura de 1990 (ITS-90).

El valor teórico definido para el coeficiente de corrección por presión hidrostática para una celda de punto triple de agua en condiciones ideales es de $-0,73 \mathrm{mK} / \mathrm{m}$ [1]. Sin embargo, se ha observado que el comportamiento real de las celdas de punto triple de agua se desvía del valor teórico, y que, para la realización del punto triple de agua, es necesario estudiar en cada celda empleada el perfil térmico correspondiente, respecto a la posición del elemento sensor en el pozo de las celdas. Es probable que el apartamiento encontrado se deba a pérdida de flujo de calor por conducción a través de la vaina del elemento sensor con el que se estén efectuando las medidas. Este trabajo se realiza para determinar las curvas de variación de temperatura del elemento sensor versus la posición en el pozo de la celda, y así poder obtener las desviaciones de la curva teórica de corrección por presión hidrostática para las celdas que se emplean como patrones nacionales en el Laboratorio Tecnológico del Uruguay, de forma de poder efectuar las correcciones necesarias. Esto tiene como consecuencia una mejor aproximación a la Escala Internacional de Temperatura ITS 90 para la calibración de termómetros de resistencia de platino (SPRT) en el punto triple de agua y la determinación de los coeficientes $\mathrm{W}$ para la calibración en los otros puntos fijos que posee el laboratorio de termometría.

\section{Materiales y Métodos}

\section{Equipos empleados}

Celda empleada. La celda empleada fue una de los dos patrones nacionales para la realización del punto triple de agua: celda de punto triple de agua marca ISOTECH modelo número BII 65 561. En la Figura 1 se muestra un esquema de la celda y del termómetro empleado y la posición de los termómetros empleados dentro de la celda.

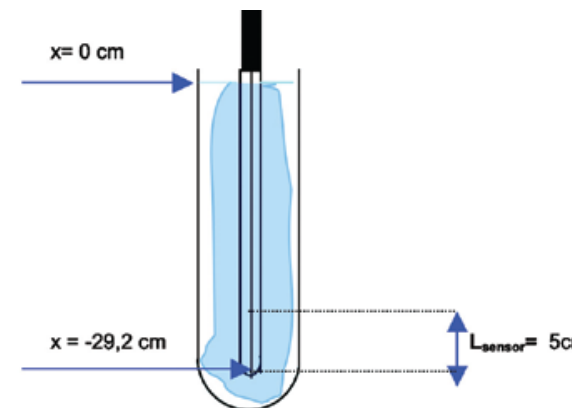

Figura 1. Representación gráfica de la celda de punto triple con SPRT. 
Se eligió en primera instancia este patrón porque es el que posee un accesorio metálico que permite eliminar el puente de hielo que se forma en la interfase sólido - líquido - vapor en la parte superior de la celda. Este puente de hielo altera el perfil térmico de la celda y se puede eliminar en forma sencilla mediante este accesorio. Por este motivo se comienza con la caracterización de la celda ISOTECH, previendo en el futuro la caracterización de la otra celda patrón disponible, CENAM 001.

Termómetros empleados. En la primera etapa se emplearon los siguientes termómetros de resistencia de platino estándar (SPRTs):

- Termómetro de resistencia de platino estándar con vaina de cuarzo marca Hart Scientific modelo 5681 número de serie 5-1055.

- Termómetro de resistencia de platino estándar con vaina de cuarzo marca Tinsley modelo $5781 \mathrm{~S}$ número de serie 274234.

- Termómetro de resistencia de platino estándar con vaina metálica marca Rosemount modelo 162 CE número de serie 3109.

Debido a los resultados obtenidos, para la segunda etapa se resolvió emplear únicamente el tercer termómetro, con el que se obtuvieron los perfiles con menor distorsión.

Esto es razonable, ya que la vaina metálica tiene conductividad térmica alta, lo que hace que los gradientes de temperatura a lo largo del termómetro correspondan a las variaciones de temperatura en el pozo de la celda.

Instrumentos de medida de resistencia. Para la medida de la relación entre la resistencia de los termómetros de platino en el punto triple y la resistencia patrón utilizada se utilizó un puente de corriente alterna marca ASL modelo F18.

La resistencia patrón empleada fue una de valor nominal $25 \mathrm{ohm}$ marca Tinsley termostatizada en un baño de aceite a $20^{\circ} \mathrm{C}$.

\section{Procedimiento experimental}

Preparación de las celdas. La celda se preenfrió en un vaso Dewar con hielo picado durante todo un día, previo a la inducción del manto de hielo introduciendo hielo seco picado $\left(\mathrm{CO}_{2}\right)$ en el pozo de la celda. La celda se mantuvo operativa por mas de tres semanas, sumergida dentro de un baño con hielo picado y aislada térmicamente en su parte superior con una tapa de material aislante y telas oscuras en ambas etapas.

En la segunda etapa se mejoró el aislamiento colocando una segunda tapa interior de material aislante.

\section{Medida del perfil térmico}

En la primera fase, para la determinación del perfil térmico se tomaron lecturas de todos los termómetros (SPRTs) a posiciones igualmente espaciadas de $1 \mathrm{~cm}$ cada una desde el fondo del pozo de la celda hasta $6 \mathrm{~cm}$ por encima. Para mover los elementos sensores se empleó un soporte metálico como guía, sujeto a la parte superior del baño de hielo utilizado.

En cada una de estas posiciones se tomaron 10 lecturas, empleando como valores de corriente $1 \mathrm{~mA}$ y $\sqrt{ } 2 \mathrm{~mA}$, para poder extrapolar los valores de la resistencia a $0 \mathrm{~mA}$. Estos últimos son los empleados para el tratamiento de datos y los cálculos.

Para determinar la incertidumbre asociada a los experimentos se realizaron otras seis series de medidas, cambiando la posición de los termómetros entre el fondo del pozo de la celda y la posición de $2 \mathrm{~cm}$ por encima del fondo en forma repetida.

Para la segunda etapa de este trabajo, y en vista de los resultados obtenidos, se preparó la celda de punto triple de la misma forma y se introdujeron alternadamente las siguientes modificaciones:
1. Agregado de una tapa adicional de espuma aislante adicional de 2 $\mathrm{cm}$ de espesor, igual diámetro que el del termo Dewar empleado.

2. Empleo de un dispositivo ecualizador de temperatura o "bushing", consistente en un tubo metálico de cobre de $5 \mathrm{~cm}$ de largo y diámetro externo inferior al diámetro interno de la celda $(10$ $\mathrm{mm}$ ), y de diámetro interno superior al diámetro externo al del termómetro de resistencia de platino empleado.

\section{Modelo matemático empleado para la expresión de los resultados}

La variación de la temperatura teórica en el punto triple de agua está expresada en la siguiente ecuación:

$t_{90}=A+B * I$

Donde:

- $\mathrm{A}=0,01{ }^{\circ} \mathrm{C}$, temperatura en el punto triple de agua

- $\mathrm{B}=$ coeficiente de corrección por presión hidrostática para el agua pura $(-0,73 \mathrm{mK} / \mathrm{m})=\mathrm{dT} / \mathrm{dh}$

- $1=$ Profundidad dentro del pozo de la celda del elemento sensor, tomando como posición nula la zona de coexistencia de las tres fases del punto triple de agua (Figura 1).

Para poder llegar a construir la curva teórica que se presenta en los gráficos de las siguientes figuras, se realizó el desarrollo que se expone a continuación:

$W_{\text {ref }}=$ polinomio correspondiente a la Escala Internacional de Temperatura de 1990 (ITS 90)

Para 273,15 K $<\mathrm{T}<1234,93 \mathrm{~K}^{(1)}$.

$$
W_{r e f}=C_{0}+\sum_{i=1}^{i=9}\left[C_{i} * \frac{\left(T_{90}-714,15\right)}{481}\right]
$$

$$
\begin{aligned}
& \text { Donde: } \\
& C_{0}=2,78157254 \\
& C_{1}=1,64650916 \\
& C_{2}=-0,13714390 \\
& C_{3}=-0,00649767 \\
& C_{4}=-0,00234444 \\
& C_{5}=-0,00511868 \\
& C_{6}=0,00187982 \\
& C_{7}=-0,00204472 \\
& C_{8}=-0,00046122 \\
& C_{9}=0,00045724
\end{aligned}
$$

La función de aproximación al polinomio de la escala ITS 90 para un termómetro de resistencia de platino estándar está dada por la ecuación:

$W_{m e d}-W_{R e f}=a^{*}\left(W_{m e d}-1\right)+b^{*}\left(W_{m e d}-1\right)^{2}$

Donde:

$$
W_{\text {med }}=\frac{R_{T}}{R_{W T P}}
$$


Corresponden al cociente las medidas efectuadas de la resistencia en el punto fijo ITS 90 en cuestión y la resistencia en punto triple de agua.

En general $a$ y $b$ son coeficientes pequeños, a los efectos de realizar las aproximaciones concernientes se puede suponer que:

$W_{\text {med }} \approx W_{\text {Ref }}$

Por consiguiente se pueden realizar las suposiciones que siguen:

$$
d W_{\text {med }}=\frac{d R_{T}}{R_{T P W}}
$$

$\frac{d T}{d h}=\frac{d W_{\text {med }}}{d h \cdot\left(\frac{d W_{\text {ref }}}{d T}\right)_{T P W}}=\frac{d R_{T}}{d h} \cdot \frac{1}{R_{T P W} \cdot\left(\frac{d W_{\text {ref }}}{d T}\right)_{T P W}}$

Luego:

$\frac{d R_{T}}{d h}=\frac{d T}{d h} \cdot\left(\frac{d W_{r e f}}{d T}\right)_{T P W} \cdot R_{T P W}$

Y la ecuación de la curva de resistencia teórica es:

$$
R_{x}=R_{x_{i}}+\frac{d R_{T}}{d h} \cdot\left(x_{f}-x_{i}\right)
$$

Donde $x_{i}=-0,265 m$ corresponde a la posición inicial del elemento sensor en el pozo de la celda y $\mathrm{xf}$ a las distintas posiciones estudiadas espaciadas cada $1 \mathrm{~cm}$, en los gráficos se toma xi como $0 \mathrm{~cm}$.

Mediante la ecuación anterior se puede construir la curva teórica de variación de la resistencia con la que se comparan los datos experimentales obtenidos.

\section{Resultados}

\section{Perfiles térmicos de la celda Isotech}

Etapa 1. En la Figuras 2, 3 y 4 se presentan las curvas de resistencia promedio versus posición del termómetro para la celda marca Isotech de los termómetros Tinsley, Hart y Rosemount, respectivamente.

La curva de resistencia vs. posición en el pozo de la celda teórica, considerando únicamente el coeficiente de corrección por presión hidrostática, está expresada en las figuras que siguen a continuación por la línea recta.

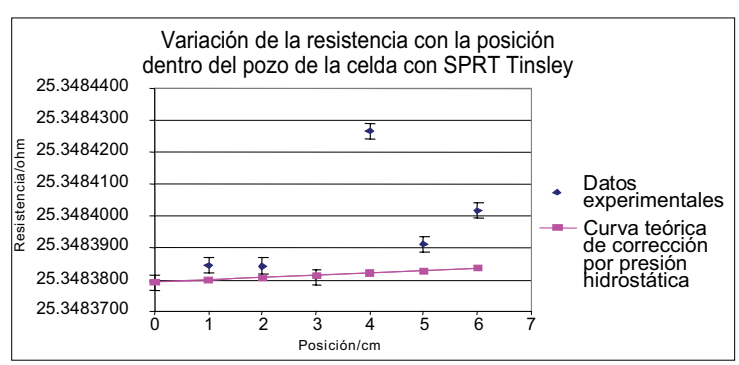

Figura 2. Curva de variación del valor de la resistencia (ohm) vs posición $(\mathrm{cm})$ desde el fondo del pozo de la celda Isotech medida con SPRT Tinsley, luego de estabilización, etapa 1.

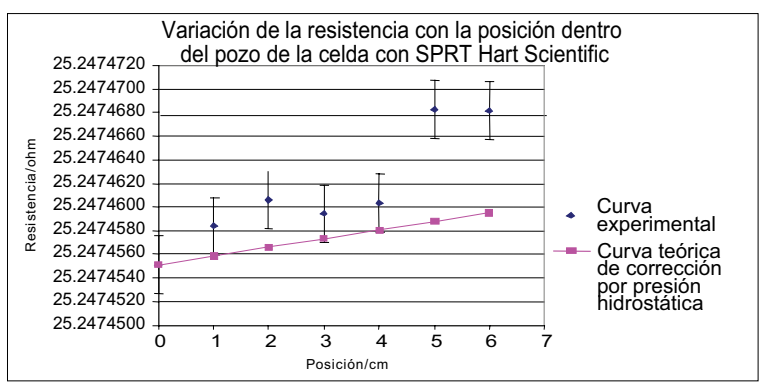

Figura 3 Curva de variación del valor de la resistencia (ohm) vs. posición $(\mathrm{cm})$ desde el fondo del pozo de la celda Isotech medida con SPRT Hart, luego de estabilización, etapa 1.



Figura 4 Curva de variación del valor de la resistencia (ohm) vs. posición $(\mathrm{cm})$ desde el fondo del pozo de la celda Isotech medida con SPRT Rosemount, luego de estabilización, etapa 1.

Si se comparan los valores obtenidos en las figuras anteriores con respecto a la curva teórica, se observa que:

1. Existen desviaciones importantes con respecto al comportamiento ideal que se espera para una celda de punto triple de agua para los tres termómetros empleados en las determinaciones.

2. El tiempo de estabilización de la celda es demasiado largo para mantener la celda en condiciones óptimas (esto significa tener un manto de hielo apropiado que cubra bien el pozo de la celda). El medio térmico del que se dispone (termo Dewar) tiene sus limitaciones con respecto a otros tipos de baños termostatizados que existen en plaza en cuanto a la transferencia térmica y la estabilidad.

Etapa 2. Introducción de tapa aislante adicional. Se comenzaron a efectuar mediciones a cuatro días de haber realizado el punto triple.

La Figura 5 muestra los resultados de la primera medición. El perfil térmico se encuentra completamente desviado con respecto al comportamiento teórico que debería presentar la celda. 


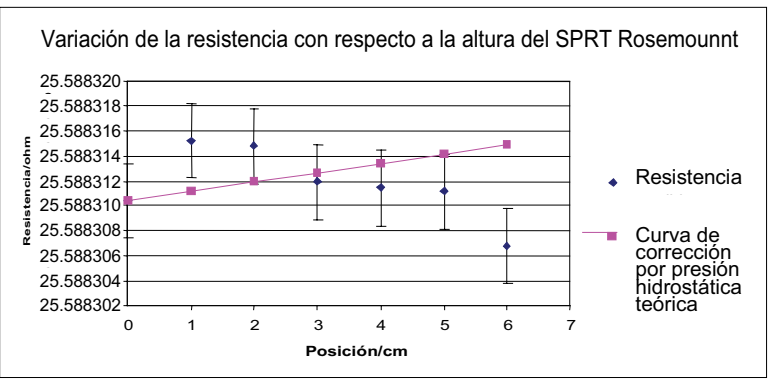

Figura 5. Curva de variación del valor de la resistencia (ohm) versus la posición $(\mathrm{cm})$ desde el fondo del pozo de la celda Isotech medida con SPRT Rosemount, luego de cuatro días de haber sido armada, etapa 2.

Si se observa la Figura 6, los datos obtenidos tres días después se acercaron bastante al comportamiento ideal, teniendo en cuenta el valor de incertidumbre asociado al método.

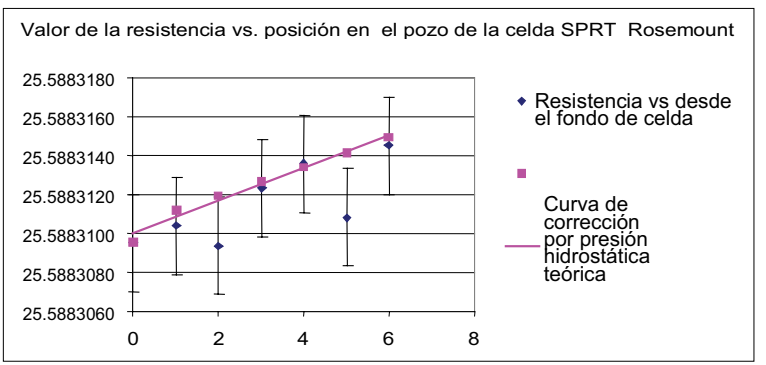

Figura 6. Curva de variación del valor de la resistencia (ohm) versus la posición $(\mathrm{cm})$ desde el fondo del pozo de la celda Isotech medida con SPRT Rosemount, etapa 2.

Las experiencias que se realizaron antes de introducir el empleo del ecualizador o bushing fueron de comportamientos similares al de la Figura 6. La Figura 7 muestra la última experiencia realizada antes de la introducción del ecualizador térmico.

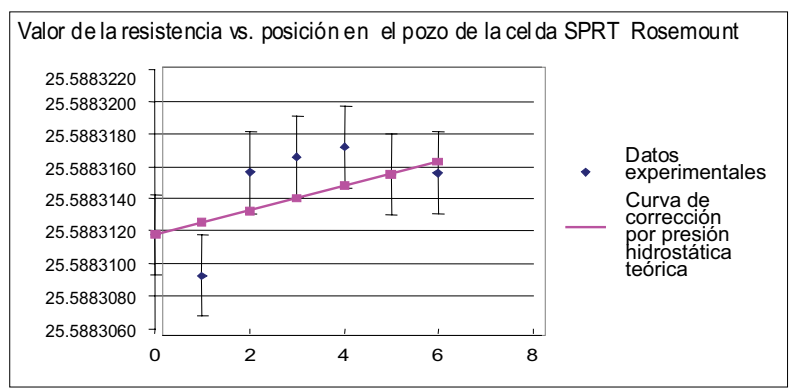

Figura 7. Curva de variación del valor de la resistencia (ohm) versus la posición $(\mathrm{cm})$ desde el fondo del pozo de la celda Isotech medida con SPRT Rosemount, última medida sin ecualizador, etapa 2.

\section{Utilización del ecualizador térmico}

Las Figuras 8, 9 y 10 muestran tres series de medidas realizadas empleando el ecualizador térmico dentro del pozo de la celda. Si bien existe una mejora en los perfiles térmicos obtenidos, ésta no es tan significativa como la de la primera modificación.

Es importante estudiar este efecto del ecualizador con el objetivo de lograr una mejor aproximación al comportamiento ideal.

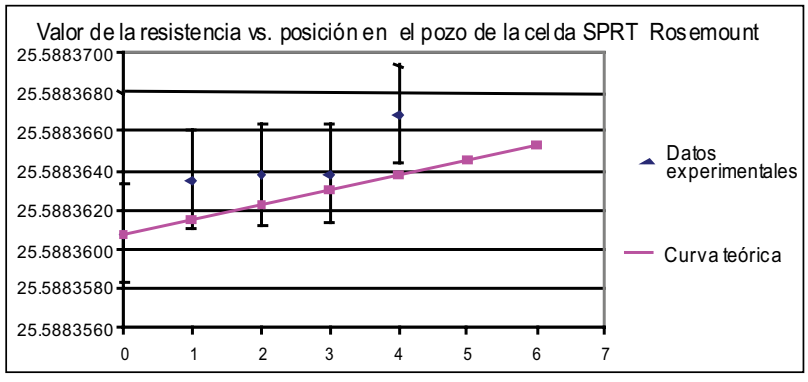

Figura 8. Curva de variación del valor de la resistencia (ohm) versus la posición $(\mathrm{cm})$ desde el fondo del pozo de la celda Isotech medida con SPRT Rosemount, primera medida con ecualizador, etapa 2.

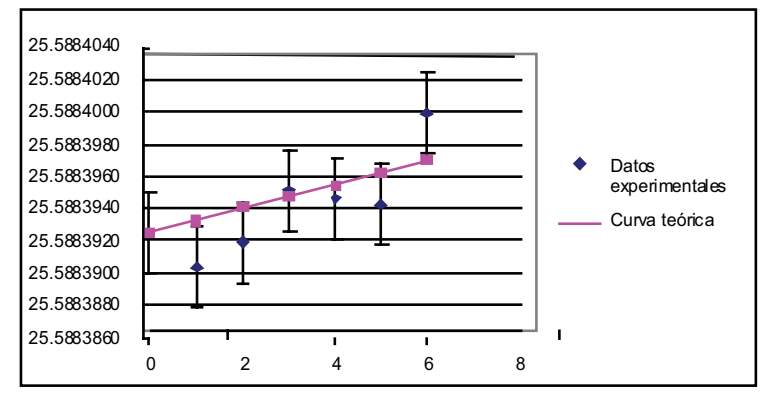

Figura 9. Curva de variación del valor de la resistencia (ohm) versus la posición $(\mathrm{cm})$ desde el fondo del pozo de la celda Isotech medida con SPRT Rosemount, segunda medida con ecualizador, etapa 2.

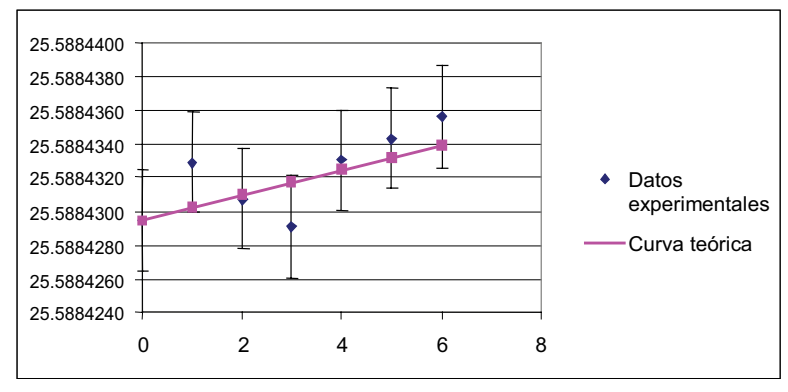

Figura 10. Curva de variación del valor de la resistencia (ohm) versus la posición $(\mathrm{cm})$ desde el fondo del pozo de la celda Isotech medida con SPRT Rosemount, tercera medida con ecualizador, etapa 2.

\section{Expresión de incertidumbre}

El valor de la incertidumbre asociada se estima a partir de la repetibilidad del ensayo, variando la posición del termómetro en foma sucesiva entre la posición $0 \mathrm{~cm}$ (fondo del pozo de la celda) y $3 \mathrm{~cm}$. No se incluyen componentes de error sistemático debido a que se calcula el valor de la pendiente de una recta empleado (se miden variaciones de temperatura y no temperaturas absolutas). Sí se toma en cuenta la división del instrumento de medición de resistencia. La incertidumbre se estima en $\pm 0,030 \mathrm{mK}$.

\section{Discusión}

\section{Discusión de los resultados para la celda Isotech en la etapa 1}

Los resultados obtenidos en el caso de los termómetros Tinsley y Hart demuestran que existe una componente de flujo de calor longitudinal hacia el interior de la celda que distorsiona el perfil térmico medido. 
El motivo del apartamiento del perfil térmico obtenido con el termómetro Tinsley es que la celda requiere de unos días de estabilización antes de comenzar a tomar mediciones.

Sin embargo, el perfil térmico de las repeticiones efectuadas para el termómetro Tinsley presenta el mismo comportamiento en los valores obtenidos de resistencia a las distintas posiciones.

Para el termómetro Hart se observa un comportamiento más regular, igualmente el apartamiento es significativo con respecto a la curva teórica. Para el termómetro Rosemount el resultado no se aparta tanto del teórico como en los casos citados anteriormente. Se esperaba que debido a que el termómetro tiene vaina metálica el flujo de calor hacia dentro de la celda fuese mayor que para los otros dos termómetros de vaina de cuarzo, ya que la conductividad térmica de un metal es mayor que la conductividad térmica del cuarzo. El mejor comportamiento puede deberse a haber logrado la estabilización de la celda.

\section{Discusión de los resultados para la celda Isotech en la etapa 2}

Los resultados obtenidos en la segunda fase a partir de las modificaciones empleadas llevaron a una mejor aproximación al comportamiento teórico de la corrección por presión hidrostática para el punto triple de agua. Esto es fundamental a la hora de realizar las correcciones correspondientes para expresar resultados en las calibraciones de termómetros de resistencia de platino. La celda se estabilizó en un tiempo menor que en la primera etapa debido a la mejora que se efectuó en la aislación. Esto llevó a que se pudieran realizar más experiencias que permitiesen confirmar la mejoría con las dos modificaciones introducidas.

\section{Conclusiones}

Existen dificultades cuando se pretende estudiar detalladamente el perfil térmico por presión hidrostática de cada celda de punto fijo (ITS90), ya que pueden hallarse desviaciones importantes con respecto al comportamiento ideal. En este caso el proceso se realizó para el punto triple de agua, pero se debe realizar el mismo análisis con el resto de las celdas patrones de puntos fijos ITS-90 que hay en el laboratorio.

Una serie de pequeñas modificaciones introducidas llevaron a resultados significativamente mejores, que permiten afirmar que la celda estudiada tiene un comportamiento que se acerca al comportamiento teórico en las condiciones de ensayo.

Lo efectuado ya con el termómetro Rosemount se debe repetir para el resto de los termómetros de resistencia de platino con vaina de cuarzo.

\section{Reconocimientos}

Se agradece a las siguientes personas por sus aportes invalorables para la realización de este trabajo:

Al Ing. Quím. Luis Mussio, por la formación inicial que nos brindó en la disciplina de Termometría y por sus comentarios rigurosos sobre el tema.

A la Dipl. Ing. Ute Noatsch, del Physikalisch Technische Bundesanstalt de Alemania (PTB), quien nos introdujo en los detalles concernientes a la calibración de termómetros estándar de resistencia de platino en puntos fijos ITS-90.

Al Dr. Steffen Rudtsch, del Physikalisch Technische Bundesanstalt de Alemania (PTB), quien hizo hincapié en la necesidad de la caracterización de los patrones que se utilizan en el laboratorio de termometría durante la Peer Review efectuada por el BIPM en el mes enero de 2008.

A Adrián Solano, del Laboratorio Costarricense de Metrología (LACOMET), quien nos sugirió el uso de ecualizadores.
A David Southworth, de Isothermal Technology Limited (ISOTECH), por la sugerencia acerca del material y de las dimensiones para la construcción del ecualizador térmico.

A Jorge Navas, del Departamento de Metrología Científica del LATU, quien suministró el material para la construcción del material del ecualizador.

\section{Referencias}

- PRESTON-THOMAS, H. The International temperature scale of 1990 (ITS-90). En: Metrologia. 1990, (27):3-10.

- SAKURAI, Hirohisa. Hydrostatic pressure correction coefficient of the triple point cell of water [En línea]. Cedrex: Bureau International des Poids et Mesures, [s.d.], [Consulta: 27 de marzo de 2008]. (CCT; 05-11). Disponible en:

http://www.bipm.org/cc/CCT/Allowed/23/CCT_05_11.pdf 